\title{
Development of Fetal Growth Charts in Twins Stratified by Chorionicity and Mode of Conception: a Retrospective Cohort Study in China
}

\author{
Yuanqing Xia ${ }^{1}$, Shuping $\mathrm{Lyu}^{2}$, Yiting $\mathrm{Chen}^{1}$, Li Gao ${ }^{2}$, Anda Zhao ${ }^{3}$, Yanlin Wang ${ }^{2}$, and \\ Shenghui $\mathrm{Li}^{4}$ \\ ${ }^{1}$ Shanghai Jiao Tong University School of Public Health \\ ${ }^{2}$ International Peace Maternity \& Child Health Hospital, Shanghai Jiao Tong University \\ School of Medicine \\ ${ }^{3}$ Shanghai Ninth People's Hospital, Shanghai Jiao Tong University School of Medicine \\ ${ }^{4}$ School of Public Health, Shanghai Jiao Tong University School of Medicine
}

August 28, 2020

\begin{abstract}
Objectives: We attempted to establish a set of fetal biometric references for Chinese twin pregnancies, stratified by chorionicity and conception mode as spontaneously conceived monochorionic diamniotic (SC-MCDA), spontaneously conceived dichorionic diamniotic (SC-DCDA) and assisted reproductive technology dichorionic diamniotic (ART-DCDA). Design: Retrospective cohort study. Setting: International Peace Maternity \& Child Health Hospital, shanghai, China. Population or Sample: 929 twin pregnant women, along with 2019 singleton pregnant women, were qualified for the inclusion criteria. Methods: The linear mixed models were used to test the difference of growth pattern between groups, and the growth curve of each biometric parameter was modeled by generalized additive model for location scale and shape. Main Outcome Measures: From 2016 to 2019, ultrasonographic fetal biometric measurements were longitudinally collected in pregnant women, in which six measurements were recorded: fetal weight, biparietal diameter, head circumference, abdominal circumference, femur length and humerus length. Results: Overally, SC-DCDA twins grew faster than SC-MCDA twins, while slower than ART-DCDA twins. Correspondingly, the week-specific differences between groups were also identified for all the six biometric measurements, though the differences were not observed in all gestational weeks. The customized fetal growth charts of each fetal biometric parameter were then respectively constructed for SC-MCDA, SC-DCDA and ART-DCDA twins, and all of the three shows significant difference from singletons, especially during the third trimester. Conclusions: The fetal biometric trajectories demonstrated characterized pattern according to chorionicity and conception mode. We are the first to curve fetal biometric reference values for Chinese SC-MCDA, SC-DCDA and ART-DCDA twin pregnancies.
\end{abstract}

\section{Introduction}

In recent years, the rate of twin pregnancies has continued to rise due to the growing utilization of assisted reproductive technology (ART) and late childbirth ${ }^{1,2}$. Available data suggest that ART accounts for a third of twin pregnancies ${ }^{3,4}$. It has been well documented that fetal growth of twin fetuses is slower than that of singletons, usually starting from 28 to 32 week of gestation ${ }^{5-8}$, owing to the limited uterine space ${ }^{9}$.

Up to date the clinical examination for the intrauterine growth of twins still largely relies on the growth standards of singletons, and it has been an increasing focus to develop twin-specific biometry chart to monitor fetal growth trajectory for twin pregnancy ${ }^{10}$. In recent years, several ultrasonographic reference charts of twins have been established ${ }^{5-8,11-16}$, however, they were derived from small populations ${ }^{5,11,15}$ or did not 
rule out high risk pregnancies ${ }^{5,12,16}$. In addition, evidence suggested that, compared with dichorionic diamniotic (DCDA) twins, monochorionic diamniotic (MCDA) twins showed a slower growth rate ${ }^{6,11,16}$, and ART may affect the perinatal outcome of twin pregnancies ${ }^{17-20}$. Therefore, both chorionicity and conception mode should be taken into consideration when developing fetal biometric reference for twins. A newly published study from Italy established the first longitudinal growth charts for fetal ultrasound biometry customized for chorionicity, however, the data didn't show statistical difference of fetal growth over gestation age between DCDA and MCDA twins ${ }^{6}$. To date, no study explored the differences of fetal intrauterine growth between ART and spontaneously conceived (SC) twin pregnancies. To fill the knowledge gap, our study would examine the growth difference of twins with varied chorionicity and conception mode, aiming to establish chorionicity- and conception mode-specific fetal biometric parameters reference.

Existing studies modeled the growth curve adopting linear mixed model ${ }^{5-7,12,13}$, multilevel linear models ${ }^{14,15}$ or hierarchical Bayesian models ${ }^{8}$, in all of which the fitting precision and accuracy were somewhat weakened by the data's deviation in skewness and kurtosis coefficient. Compared to linear model, the generalized additive model for location, scale and shape (GAMLSS) extends to model all the fourth-order variations, including median, standard deviation, skewness, and kurtosis, demonstrating a strong strength in improving the accuracy of fitting smoothed percentile curves ${ }^{21}$. Since 2006, WHO performed GAMLSS to establish child growth standards ${ }^{10}$.

The fetal growth can be differed by race or ethnicity ${ }^{22,23}$. In 2015 , a Chinese study initially established a standard for twin fetal weight growth ${ }^{24}$. However, the study was based on birth weight data but not ultrasonographic biometric parameters, while the birth weight data can be biased by preterm delivery since preterm delivery is usually associated with pregnancy complications and fetal growth abnormalities. Therefore, the standard established in this study somewhat sacrificed the sensitivity to identify the early onset of growth restriction and cannot convey the longitudinal pattern of fetal growth from early pregnancy ${ }^{24}$.

The present study would step forward to fit GAMLSS model based longitudinal growth trajectories among Chinese pregnant women by using ultrasonographic biometry data. We are the first to develop fetal growth reference for Chinese twin pregnancies stratified by both chorionicity and mode of conception, and the reference would be tested through the comparison with data from singletons.

\section{Methods}

\subsection{Study Population}

Based on fetal ultrasonographic biometry electronic datasets, a retrospective longitudinal study was conducted among pregnant women from the prenatal diagnosis department of International Peace Maternity \& Child Health Hospital, Shanghai, China. Those pregnant women who delivered twins as well as singleton between January 2016 and December 2019 were included.

This study would attempt to construct ultrasonic biometry standards to monitor fetal growth for twin pregnancy, only delivery at or beyond 34 weeks of gestation, along with at least two sets of measurements during the whole pregnancy, were considered to be qualified. Exclusion criteria were listed as the followed: uncertain chorionicity; monoamnionicity; key information deficit, mainly gestational age or ultrasonic measurements being unavailable; spontaneous or iatrogenic reduction from a multifetal gestation; fetal death; fetal structural or chromosomal anomalies; occurrence of twin-to-twin transfusion syndrome or twin anemiapolycytemia sequence; selective fetal growth restriction or a birthweight below the 3th percentile for the national birthweight charts ${ }^{25}$; preexisting maternal disease such as hypertension, diabetes, or renal and autoimmune disorders; and the development of obstetric complications such as gestational hypertensive disease and diabetes. In addition, only very minority $(\mathrm{n}=23)$ was MCDA twins conceived by ART, therefore, they were not included in the final analyses; and this study focused on spontaneously conceived monochorionic diamniotic (SC-MCDA), spontaneously conceived dichorionic diamniotic (SC-DCDA) and assisted reproductive technology dichorionic diamniotic (ART-DCDA). While for singleton pregnancy, only delivery at or beyond 37 weeks of gestation were selected, and the inclusion/exclusion criteria were defined in accordance with those of twin pregnancy where appropriate. 
Gestational age was calculated by the date of ovulation, the date of embryo transfer, last menstrual period, or the crown-rump length, as appropriate. The diagnosis of chorionicity was based on the number of gestational sacs at 7-8 weeks of gestation and "T sign", or "lambda sign" obtained by ultrasonography at the first trimester.

Maternal age at delivery was grouped into two categories: $<35$ vs. [?]35years, and [?]35years was defined as advanced maternal age. Maternal prepregnancy body mass index was grouped into four categories: $<18.5$, 18.5-23.9, 24.0-27.9, and [?]28.8.

\subsection{Fetal Ultrasonic Measurements}

Ultrasound examinations were performed every 3-4 weeks between 14 and 32 weeks, then every 2 weeks until delivery in singleton and DCDA twins; while for MCDA twins, every 2 weeks between 14 and 32 weeks and weekly beyond 32 weeks of gestation. At each visit, the fetal biparietal diameter (BPD), head circumference (HC), femur length (FL), humerus length (HL), anteropostero trunk diameter (APTD) and transverse trunk diameter (TTD) for each fetus were measured and recorded. The BPD was measured from the outer to the inner edge of the fetal skull at the level of the thalami. The HC was measured as an ellipse around the perimeter of fetal skull. The FL was measured from one end of the femoral diaphysis to the other, not including the distal femoral epiphysis. The HL was measured from one end of the humeral diaphysis to the other with the borders clearly visible. Both APTD and TTD extended from the outer aspects of the lateral abdominal wall, and TTD usually was perpendicular to APTD. Estimated fetal weight (EFW) was calculated based on BPD, APTD, TTD and FL according to Hadlock formulas ${ }^{26,27}$. Abdominal circumference (AC) was calculated based on the mean arithmetic diameters of APTD and TTD ${ }^{28}$.

\subsection{Statistical analysis}

Statistical description was made by use of percentage for categorical variables, and mean, along with standard deviation, for continuous variables. The group difference was examined using Chi-squared test, variance analysis, or Kruskal-Wallis rank test where appropriate, and the pairwise comparisons were further checked.

The linear mixed models were used to test the fetal growth difference by varied chorionicity and mode of conception. Both the overall longitudinal change including trajectory curve and growth velocity across the pregnancy and week-specific discrepancy were taken into examination. For the purpose of this study, the difference was compared between any two groups. For those ultrasound measurements which showed statistically different either between SC-MCDA and SC-DCDA twins or between SC-DCDA and ARTDCDA twins, week-specific comparison were further conducted after adjusting for maternal age, height, weight, gravidity, and parity ${ }^{5,6}$. Moreover, all of the three groups were in comparison with uncomplicated singletons, which delivered in the same hospital at the same time.

The GAMLSS was applied to fit the growth curve of each biometric parameter in relation to gestational age. The GAMLSS is highly flexible as it is capable of modeling not only mean (or location) but also other parameters (such as standard deviation, skewness and kurtosis) ${ }^{10,21}$. Box-Cox t (BCT) distribution was used for modeling fetal biometric parameters as non-parametric cubic spline functions of gestational age. Model selection was based on generalized Akaike Information Criterion (GAIC), and the model with the smallest value of GAIC was selected. Worm plots were used for visual inspection of the fit of the smoothed curves. Centile curves for each biometric parameter were constructed according to placental chorionicity and mode of conception.

All analyses were performed with the Statistical Package for the Social Sciences version 24 (SPSS Inc, Chicago, IL) and the GAMLSS and lme4 package for R statistical software (version 3.5.1). Significance was defined as a 2 -tail probability value of $<0.05$.

\section{Results}

A total of 4055 pregnancy women, 2142 singleton vs. 1913 twin, were recruited in this study. The flowchart of twin participants enrollment was shown in Figure 1 (The enrollment flowchart for singleton participants 
is very similar and not shown). The final sample consisted of 2019 singleton, alongside with a total of 9787 ultrasound observations, and 929 mothers (148 SC-MCDA, 215 SC-DCDA and 566 ART-DCDA), alongside with a total of 12837 ultrasound observations (3099 in 296 SC-MCDA, 2542 in 430 SC-DCDA and 7196 in 1132 ART-DCDA fetuses). A gestational age interval between 14 and 37 weeks was covered.

\subsection{Characteristics of the study group}

Description and comparison of the maternal and neonatal characteristics between SC-MCDA, SC-DCDA and ART-DCDA twins are displayed in Table S1 . Women in advanced maternal age accounted for 15.5\% among SC-MCDA twins, $19.5 \%$ in SC-DCDA twins and $24.2 \%$ in ART-DCDA twins $(p<0.001)$. Maternal prepregnancy body mass index showed significant differences only between SC-MCDA and ART-DCDA twins $(p<0.05)$. In addition, the proportion of nulliparous women in ART-DCDA twins were higher than those in SC-MCDA twins $(p<0.05)$. SC-MCDA twins had a shorter gestational age than SC-DCDA and ART-DCDA twins $(p<0.05)$. Meanwhile, SC-MCDA twins were significantly lighter in birthweight and shorter in birth length than SC-DCDA and ART-DCDA twins $(2468.82 \mathrm{~g}$ vs $2643.62 \mathrm{~g}$ and $2637.71 \mathrm{~g} ; 47.21 \mathrm{~cm}$ vs 47.82 and $47.89 \mathrm{~cm}$; all $p<0.05)$. The description of singleton and comparison with twins are displayed in Table S2 .

\subsection{Modeling fetal growth reference}

As shown in Table S3, S4 and S5, both the overall growth curve through 14 to 37 weeks of gestation and each fetal biometric measurement according to gestational age were compared among the three groups. The statistical differences occurred in the fetal biometric measurement curves for EFW, AC, FL and HL between SC-DCDA and SC-MCDA twins, and the size of each biometric measurement appeared to be smaller in SC-MCDA (all $p<0.05)$. The growth velocity for EFW, BPD, HC, FL and HL of SC-DCDA twins was also different from those of SC-MCDA twins (all $p<0.05$ ), but no statistical differences were found in AC. When evaluating week-specific differences in the biometric variables between SC-MCDA and SC-DCDA twins, the results presented that EFW, FL and HL growth slowed down in SC-MCDA twins compared with SC-DCDA during most of the gestational weeks $(p<0.05)$. In addition, the size of all the six fetal biometric measurements demonstrated smaller through 15, 21 and 25 weeks of gestation in SC-MCDA twins than that in SC-DCDA twins consistently $(p<0.05)$. When the comparison between SC-DCDA and ART-DCDA groups was examined, significant differences were observed in all of the six fetal biometric measurements curves and only in the growth velocity for $\mathrm{HC}$ (all $p<0.05$ ). Similarly, week-specific differences of all the six fetal biometric measurements were significant at a few weeks of gestation (all $p<0.05$ ). Further focusing on SC-MCDA and ART-DCDA twins, it was found that there were significant differences in all of the six fetal biometric measurements curves and the growth velocity of EFW, BPD, HC, FL and HL. Meanwhile, week-specific differences of all the six fetal biometric measurements were statistically significant during most gestational weeks.

Tables S6, S7 and S8 showed that all of the SC-MCDA, SC-DCDA and ART-DCDA twins were different from singletons, especially during the third trimester.

Figure 2, 3 and 4 show the fitted growth curves of fetal biometric parameters, including EFW, BPD, HC, AC, FL and HL for SC-MCDA, SC-DCDA and ART-DCDA twins, respectively. The smoothed percentiles, such as the 5th, 10th, 50th, 90th, and 95th percentiles of fetal biometric parameters by gestational age are illustrated in Table S9 .

\section{Discussion}

\subsection{Main findings}

Based on a large data set of ultrasonographic biometric measurements, we are the first to develop fetal ultrasound biometric standards, including EFW, BPD, HC, AC, FL and HL, among Chinese uncomplicated twin pregnancies. The major strength of this study lies in taking both chorionicity and mode of conception into account, and the findings showed that SC-DCDA twins had faster growth rate than SC-MCDA twins, while slower than ART-DCDA twins. When making comparison with data from singletons, all of the three 
shows slower growth pattern, however, the contrasts were mainly observed during the third trimester. The data enriched our understanding on the twin-specific fetal growth trajectories, and the standards established in this study have potential to promote more accurate assessment of intrauterine growth trajectory for Chinese twins.

\subsection{Interpretation}

In our study, it was demonstrated that the fetal biometric measurements including EFW, AC, FL and HL of SC-DCDA twins were statistically different from those of SC-MCDA twins. To date a few number of studies compared intrauterine growth pattern of DCDA twins to that of MCDA twins ${ }^{6-8,11-13,16}$, and the results were generally consistent and similar to the findings of our study. Among which, a study in Brazil got almost the same result with ours that there were statistically differences in EFW, BPD, AC and FL between monochorionic and dichorionic twin pregnancies ${ }^{11}$. A multicentric study from Italy also reported that the measurements of EFW, BPD, HC, AC and FL appeared smaller in MCDA group in comparison with DCDA twins, however, the difference didn't show statistical significance ${ }^{6}$. When further exploring the week-specific difference for fetal biometric measurements between DCDA and MCDA twins, a study conducted in the US found that DCDA twins had higher sonographic EFW at almost all gestational ages until 34 gestational weeks ${ }^{13}$, and the data from Italy similarly found that the differences for AC were statistically significant after 33 week of gestation ${ }^{6}$. The evidence from the two studies provided support for our findings.

Fetal growth velocity has been identified as an important indicator to assess fetal growth and development ${ }^{29}$. Recently, a national study in the US examined the growth velocity of six parameters including EFW, BPD, $\mathrm{HC}, \mathrm{AC}, \mathrm{HL}$ and FL among singletons and compared the overall differences of these parameters between different racial/ethnic-specific curves ${ }^{30}$. To our knowledge, no study yet paid attention to the fetal growth velocity among twins. ART has become more common, and an understanding with regard to it's perinatal outcome is becoming essential ${ }^{17-20}$. Several studies have, more often than not, focused on the possible impact of ART on birth weight among twin pregnancies, failing to cover intrauterine fetal growth in the concern ${ }^{17-20}$. As an initial study, we, for the first time, observed the gestational week-specific fetal size and growth velocity among ART-DCDA twins, revealing ART-DCDA twins had the highest growth rate, followed by SC-DCDA, and then SC-MCDA.

\subsection{Clinical implications}

The accuracy of intrauterine growth assessment for twins depends on the establishment of twin-specific growth charts, and the longitudinal ultrasonographic standards have benefits in recognizing growth pattern variations at different gestational ages ${ }^{31}$. The international society of obstetrics and gynecology ultrasound emphasized the clinical significance of development and use of twin-specific growth charts when assessing fetal growth of twins since $2016^{32}$. In medical practice, it has been well-known that growth restriction in twins is prevalent due to the slower growth rate in the third trimester ${ }^{5-8}$. In our study, all the median (50th percentile) biometric parameters of SC-MCDA, SC-DCDA, ART-DCDA twins were lower at each gestational week compared with fetal biometry reference of Chinese singleton ${ }^{33,34}$. Therefore, over-diagnosis of restricted intrauterine growth should be a common concern when adopting the diagnostic criteria based upon the standard of singletons. For a long time, although there are the continuous efforts, trying to establish fetal growth curve for twins, but the issue has not been resolved. The existing data has inherent limitations, for example, some of the data came from birth weight and cannot be applied to the growth and development assessment during pregnancy ${ }^{24,35-38}$, some others recruited subjects by small sample size ${ }^{5,11,15}$ or without exclusion of high-risk pregnancy ${ }^{5,12,16}$. In the present study, we got over the limitations and largely promised our growth reference chart customized for chorionicity and mode of conception a more reliable tool to distinguish cases with fetal growth restriction in twins ${ }^{39}$.

We are aware that downgrading the fetal growth reference may sacrifice the sensitivity to identify pathological fetal growth restriction. Our subject enrollment strategy adopted strict inclusion criteria for pregnant woman, which ensured a qualified sensitivity in the early screening of fetal growth restriction. Notably, our sampled population included pregnancies via ART. Since twin pregnancies via ART accounts for more than half 
of twins, our growth charts customized for conception mode would be more generalizable to the current population of twins in China. The results of this study illustrated an asymmetric pattern of growth velocity between DCDA and MCDA twins, which has been confirmed by previous studies ${ }^{6,11,16}$. In addition, it has been suggested that chorionicity has a significant independent effect on birthweight ${ }^{40}$, and the threshold of physiological intertwin size discordance of fetal biometry also vary according to the chorionicity ${ }^{41}$. In this case, developing growth reference charts customized for chorionicity is necessary.

In this study, six fetal biometric measurements obtained from ultrasound were opted to customize the growth reference, but not only the EFW. A previous study found that some biometric measurements may vary according to parental ethnicity or different constitutional characteristics, not all the differences can be specifically explained by the changes of $\mathrm{EFW}^{6}$. As proposed by previous studies ${ }^{42,43}$, we developed all fetal biometric parameters growth charts rather than only EFW in most studies ${ }^{8,12,13}$. The full-spectrum parameters could enrich our knowledge on fetal changes in uterus, which should be clinically significant to promote a more comprehensive evaluation of fetal intrauterine growth.

\subsection{Strengths and limitations}

The main strength of our study lies in the following points. The first one is that only healthy uncomplicated twin pregnancies were included. Secondly, our reference charts were based on longitudinal data as well adequate sample size, which made it available to develop growth centiles to identify patterns and differences in fetal growth at different gestational ages ${ }^{14}$. Thirdly, taking both chorionicity and mode of conception into account promised our growth reference chart a more reliable and sensitive tool in identifying growth restriction in twins. Forth, the GAMLSS model has unique advantages in fitting the changing curve over time, which has been applied by WHO to establish child growth standards ${ }^{10}$. Moreover, making comparison with data from singleton provided evidence to confirm that twin has intrinsic growth pattern, emphasizing the importance of this study.

However, several weaknesses also warrant acknowledgement. First, this study was a single-center design, there might be a selection bias. However, International Peace Maternity \& Child Health Hospital is the top quality maternal and child health hospital in Shanghai, which attracts pregnant women all around Shanghai. The hospital has opened twin pregnancy clinic since 2015, which is one of three specialist consultation settings for twin pregnancy in Shanghai. Therefore, the twin pregnant women recruited through four years should have a certain representative in Shanghai area. Secondly, very minority $(n=23)$ was MCDA twins conceived by ART, therefore, they were not included in this study. Finally, although we adopted strict inclusion criteria to acquire qualified target population, other high-risk individual with unclear adverse perinatal outcomes was possible.

\section{Conclusion}

For the first time, we established a set of fetal biometric parameter references stratified by chorionicity and mode of conception for Chinese twins, and the clinical significance lies in providing a useful tool for more accurate and comprehensive assessment of fetal growth in twins. This study provides new evidence that SC-MCDA twins grow slower than SC-DCDA twins, and the growth velocity of EFW, BPD, HC, FL and HL were statistically different between them. Meanwhile, our results showed that conception mode could affect the growth pattern of twins.

\section{Abbreviations:}

EFW, estimated fetal weight; BPD, biparietal diameter; HC, head circumference; AC, abdominal circumference; FL, femur length; HL, humerus length; ART, assisted reproductive technology; SC, spontaneously conceived; DCDA, dichorionic diamniotic; MCDA, monochorionic diamniotic; SC-MCDA, spontaneously conceived monochorionic diamniotic; SC-DCDA, spontaneously conceived dichorionic diamniotic; ART-DCDA, assisted reproductive technology dichorionic diamniotic

\section{Declarations:}




\section{Acknowledgments}

We would like to express our gratitude to all the women who participated in this study, as well as all the doctors and nurses who assisted with data collection for this research.

\section{Ethical Statement}

The ethical application and consent procedure were approved by the Ethics Committee of Shanghai Jiao Tong University School of Medicine (Approval number: SJUPN-201717, dated 28 December 2017). As this is a retrospective study, informed consent is not applicable.

\section{Consent for publication}

Not applicable.

\section{Availability of data and material}

The database used for the study is not public but it is available from the corresponding author on reasonable request.

\section{Conflict of interests}

The authors declare that they have no competing interests.

\section{Funding}

The study was funded by grants from National Natural Science Foundation of China $(81673183,81874266)$, and the Clinical Guidance Project of Shanghai Science and Technology Commission (164119622800).

\section{Author Contributions}

Yuanqing Xia: Project development, Data analysis, Manuscript writing, Critical revision of the manuscript; Shuping Lyu: Project development, Data collection or management, Critical revision of the manuscript; Yiting Chen: Manuscript writing, Critical revision of the manuscript; Li Gao: Data collection or management; Anda Zhao: Data analysis; Yanlin Wang: Project development, Data collection or management, Critical revision of the manuscript; Shenghui Li: Project development, Manuscript writing, Critical revision of the manuscript.

\section{Reference:}

1. J. Smits and C. Monden. Twinning across the Developing World. PloS one. 2011; 6: e25239.

2. C. V. Ananth and S. P. Chauhan. Epidemiology of twinning in developed countries. Seminars in perinatology. 2012; 36: 156-61.

3. C. Calhaz-Jorge, C. de Geyter, M. S. Kupka, J. de Mouzon, K. Erb, E. Mocanu, et al. Assisted reproductive technology in Europe, 2012: results generated from European registers by ESHRE. Human reproduction (Oxford, England). 2016; 31: 1638-52.

4. P. Gill and M. J. Van Hook. Twin Births. In StatPearls . StatPearls Publishing LLC.: Treasure Island (FL), 2019.

5. K. L. Grantz, J. Grewal, P. S. Albert, R. Wapner, M. E. D'Alton, A. Sciscione, et al. Dichorionic twin trajectories: the NICHD Fetal Growth Studies. Am J Obstet Gynecol. 2016; 215: 221 e1- e16.

6. T. Ghi, F. Prefumo, A. Fichera, M. Lanna, E. Periti, N. Persico, et al. Development of customized fetal growth charts in twins. Am J Obstet Gynecol. 2017; 216: 514.e1-.e17.

7. L. Hiersch, R. Okby, H. Freeman, H. Rosen, O. Nevo, J. Barrett, et al. Differences in fetal growth patterns between twins and singletons $(*)$. The journal of maternal-fetal \& neonatal medicine : the official journal of the European Association of Perinatal Medicine, the Federation of Asia and Oceania Perinatal Societies, the International Society of Perinatal Obstet. 2019: 1-10. 
8. M. Sekiguchi, M. Mikami, C. Nakagawa, M. Ozaki, S. Tanigaki, T. Kobayashi, et al. An ultrasonographic estimated fetal weight reference for Japanese twin pregnancies. Journal of medical ultrasonics (2001). 2019; 46: $209-15$.

9. E. J. Hayes. Review of "Multiple Pregnancy: Epidemiology, Gestation \& Perinatal Outcome. Second Edition”. Journal of Experimental \& Clinical Assisted Reproduction. 2005; 2: 1-3.

10. W. M. G. R. S. Group. WHO Child Growth Standards based on length/height, weight and age. Acta paediatrica (Oslo, Norway : 1992) Supplement. 2006; 450: 76-85.

11. E. Araujo Junior, R. Ruano, P. Javadian, W. P. Martins, J. Elito, Jr., C. R. Pires, et al. Reference charts for fetal biometric parameters in twin pregnancies according to chorionicity. Prenatal diagnosis. 2014; 34: $382-8$.

12. S. Shivkumar, K. P. Himes, J. A. Hutcheon and R. W. Platt. An ultrasound-based fetal weight reference for twins. Am J Obstet Gynecol. 2015; 213: 224.e1-9.

13. R. Gabbay-Benziv, S. Crimmins and S. A. Contag. Reference Values for Sonographically Estimated Fetal Weight in Twin Gestations Stratified by Chorionicity: A Single Center Study. Journal of ultrasound in medicine : official journal of the American Institute of Ultrasound in Medicine. 2017; 36: 793-8.

14. X. Torres, M. Bennasar, E. Eixarch, C. Rueda, A. Gonce, M. Munoz, et al. Gender-Specific Antenatal Growth Reference Charts in Monochorionic Twins. Fetal diagnosis and therapy. 2018; 44: 202-9.

15. A. W. Liao, L. Brizot Mde, H. J. Kang, R. A. Assuncao and M. Zugaib. Longitudinal reference ranges for fetal ultrasound biometry in twin pregnancies. Clinics (Sao Paulo, Brazil). 2012; 67: 451-5.

16. O. T. Stirrup, A. Khalil, F. D'Antonio and B. Thilaganathan. Fetal growth reference ranges in twin pregnancy: analysis of the Southwest Thames Obstetric Research Collaborative (STORK) multiple pregnancy cohort. Ultrasound in obstetrics \& gynecology : the official journal of the International Society of Ultrasound in Obstetrics and Gynecology. 2015; 45: 301-7.

17. O. Shebl, T. Ebner, A. Sir, M. Sommergruber and G. Tews. The role of mode of conception in the outcome of twin pregnancies. Minerva ginecologica. 2009; 61: 141-52.

18. E. Vasario, V. Borgarello, C. Bossotti, E. Libanori, M. Biolcati, S. Arduino, et al. IVF twins have similar obstetric and neonatal outcome as spontaneously conceived twins: a prospective follow-up study. Reproductive biomedicine online. 2010; 21: 422-8.

19. X. Yang, Y. Li, C. Li and W. Zhang. Current overview of pregnancy complications and live-birth outcome of assisted reproductive technology in mainland China. Fertility and sterility. 2014; 101: 385-91.

20. J. B. Qin, H. Wang, X. Sheng, Q. Xie and S. Gao. Assisted reproductive technology and risk of adverse obstetric outcomes in dichorionic twin pregnancies: a systematic review and meta-analysis. Fertility and sterility. 2016; 105: 1180-92.

21. D. M. Stasinopoulos and R. A. Rigby. Generalized Additive Models for Location Scale and Shape (GAMLSS) in R. Journal of Statistical Software. 2007; 23: 1-43.

22. A. Madan, S. Holland, J. E. Humbert and W. E. Benitz. Racial differences in birth weight of term infants in a northern California population. Journal of perinatology : official journal of the California Perinatal Association. 2002; 22: 230-5.

23. G. M. Buck Louis, J. Grewal, P. S. Albert, A. Sciscione, D. A. Wing, W. A. Grobman, et al. Racial/ethnic standards for fetal growth: the NICHD Fetal Growth Studies. Am J Obstet Gynecol. 2015; 213: 449.e1-.e41.

24. J. Zhang, R. Mikolajczyk, X. Lei, L. Sun, H. Yu and W. Cheng. An adjustable fetal weight standard for twins: a statistical modeling study. BMC medicine. 2015; 13: 159. 
25. L. Zhu, R. Zhang, S. Zhang, W. Shi, W. Yan, X. Wang, et al. [Chinese neonatal birth weight curve for different gestational age]. Zhonghua er ke za zhi = Chinese journal of pediatrics. 2015; 53: 97-103.

26. N. Shinozuka, T. Okai, S. Kohzuma, M. Mukubo, C. T. Shih, T. Maeda, et al. Formulas for fetal weight estimation by ultrasound measurements based on neonatal specific gravities and volumes. Am J Obstet Gynecol. 1987; 157: 1140-5.

27. F. P. Hadlock, R. B. Harrist, R. S. Sharman, R. L. Deter and S. K. Park. Estimation of fetal weight with the use of head, body, and femur measurements-a prospective study. Am J Obstet Gynecol. 1985; 151: 333-7.

28. R. K. Tamura, R. E. Sabbagha, W. H. Pan and N. Vaisrub. Ultrasonic fetal abdominal circumference: comparison of direct versus calculated measurement. Obstet Gynecol. 1986; 67: 833-5.

29. R. L. Deter, W. Lee, L. Yeo, O. Erez, U. Ramamurthy, M. Naik, et al. Individualized growth assessment: conceptual framework and practical implementation for the evaluation of fetal growth and neonatal growth outcome. Am J Obstet Gynecol. 2018; 218: S656-s78.

30. K. L. Grantz, S. Kim, W. A. Grobman, R. Newman, J. Owen, D. Skupski, et al. Fetal growth velocity: the NICHD fetal growth studies. Am J Obstet Gynecol. 2018; 219: 285.e1-.e36.

31. S. L. Johnsen, S. Rasmussen, T. Wilsgaard, R. Sollien and T. Kiserud. Longitudinal reference ranges for estimated fetal weight. Acta obstetricia et gynecologica Scandinavica. 2006; 85: 286-97.

32. A. Khalil, M. Rodgers, A. Baschat, A. Bhide, E. Gratacos, K. Hecher, et al. ISUOG Practice Guidelines: role of ultrasound in twin pregnancy. Ultrasound in obstetrics \& gynecology : the official journal of the International Society of Ultrasound in Obstetrics and Gynecology.2016; 47: 247-63.

33. Y. Xu, N. Lek, Y. B. Cheung, A. Biswas, L. L. Su, K. Y. Kwek, et al. Unconditional and conditional standards for fetal abdominal circumference and estimated fetal weight in an ethnic Chinese population: a birth cohort study. BMC pregnancy and childbirth.2015; 15: 141.

34. Y. Zhang, H. Meng, Y. Jiang, Z. Xu, Y. Ouyang, S. Li, et al. Chinese fetal biometry: reference equations and comparison with charts from other populations. The journal of maternal-fetal \& neonatal medicine : the official journal of the European Association of Perinatal Medicine, the Federation of Asia and Oceania Perinatal Societies, the International Society of Perinatal Obstet. 2019; 32: 1507-15.

35. C. V. ANANTH, A. M. VINTZILEOS, S. SHEN-SCHWARZ, J. C. SMULIAN and Y.-L. LAI. Standards of Birth Weight in Twin Gestations Stratified by Placental Chorionicity. 1998.

36. L. Dai, C. Deng, Y. Li, L. Yi, X. Li, Y. Mu, et al. Population-based birth weight reference percentiles for Chinese twins. Ann Med.2017; 49: 470-8.

37. P. Premkumar, B. Antonisamy, J. Mathews, S. Benjamin, A. Regi, R. Jose, et al. Birth weight centiles by gestational age for twins born in south India. BMC pregnancy and childbirth. 2016; 16: 64 .

38. B. Zhang, Z. Cao, Y. Zhang, C. Yao, C. Xiong, Y. Zhang, et al. Birthweight percentiles for twin birth neonates by gestational age in China. Scientific reports. 2016; 6: 31290 .

39. A. O. Odibo, A. G. Cahill, K. R. Goetzinger, L. M. Harper, M. G. Tuuli and G. A. Macones. Customized growth charts for twin gestations to optimize identification of small-for-gestational age fetuses at risk of intrauterine fetal death. Ultrasound in obstetrics \& gynecology : the official journal of the International Society of Ultrasound in Obstetrics and Gynecology. 2013; 41: 637-42.

40. A. T. Papageorghiou, V. Bakoulas, N. J. Sebire and K. H. Nicolaides. Intrauterine growth in multiple pregnancies in relation to fetal number, chorionicity and gestational age. Ultrasound in obstetrics \& gynecology : the official journal of the International Society of Ultrasound in Obstetrics and Gynecology. 2008; 32: 890-3. 
41. O. T. Stirrup, A. Khalil, F. D'Antonio and B. Thilaganathan. Patterns of Second- and Third-Trimester Growth and Discordance in Twin Pregnancy: Analysis of the Southwest Thames Obstetric Research Collaborative (STORK) Multiple Pregnancy Cohort. Fetal diagnosis and therapy. 2017; 41: 100-7.

42. M. W. Pang, T. N. Leung, D. S. Sahota, T. K. Lau and A. M. Chang. Customizing fetal biometric charts. Ultrasound in obstetrics \& gynecology : the official journal of the International Society of Ultrasound in Obstetrics and Gynecology. 2003; 22: 271-6.

43. S. L. Johnsen, T. Wilsgaard, S. Rasmussen, R. Sollien and T. Kiserud. Longitudinal reference charts for growth of the fetal head, abdomen and femur. European journal of obstetrics, gynecology, and reproductive biology. 2006; 127: 172-85.

Figure legends

Figure 1 Flowchart of study population selection.

Figure 2 Estimated percentiles for EFW, BPD, HC, AC, FL and HL in SC-MCDA twins.

EFW, estimated fetal weight; BPD, biparietal diameter; HC, head circumference; AC, abdominal circumference; FL, femur length; HL, humerus length; SC-MCDA, spontaneously conceived monochorionic diamniotic.

Figure 3 Estimated percentiles for EFW, BPD, HC, AC, FL and HL in SC-DCDA twins.

EFW, estimated fetal weight; BPD, biparietal diameter; HC, head circumference; AC, abdominal circumference; FL, femur length; HL, humerus length; SC-DCDA, spontaneously conceived dichorionic diamniotic.

Figure 4 Estimated percentiles for EFW, BPD, HC, AC, FL and HL in ART-DCDA twins.

EFW, estimated fetal weight; BPD, biparietal diameter; HC, head circumference; AC, abdominal circumference; FL, femur length; HL, humerus length; ART-DCDA, assisted reproductive technology dichorionic diamniotic.

\section{Supporting Information}

Table S1. Description and comparison of maternal and neonatal characteristics between SC-MCDA, SCDCDA and ART-DCDA twins.

Table S2. Description and comparison of maternal and neonatal characteristics between singletons and twins.

Table S3. Statistical significance for overall differences of trajectories and the growth velocity and weekspecific differences for fetal biometric parameters between SC-MCDA and SC-DCDA twins.

Table S4. Statistical significance for overall differences of trajectories and the growth velocity and weekspecific differences for fetal biometric parameters between SC-DCDA.

Table S5. Statistical significance for overall differences of trajectories and the growth velocity and weekspecific differences for fetal biometric parameters between SC-MCDA and ART-DCDA twins.

Table S6. Statistical significance for overall differences of trajectories and the growth velocity and weekspecific differences for fetal biometric parameters between singletons and ART-DCDA twins.

Table S7. Statistical significance for overall differences of trajectories and the growth velocity and weekspecific differences for fetal biometric parameters between singletons and SC-DCDA twins.

Table S8. Statistical significance for overall differences of trajectories and the growth velocity and weekspecific differences for fetal biometric parameters between singletons and SC-MCDA twins.

Table S9. Reference values for fetal biometric parameters by SC-MCDA vs. SC-DCDA vs. ART-DCDA twins. 


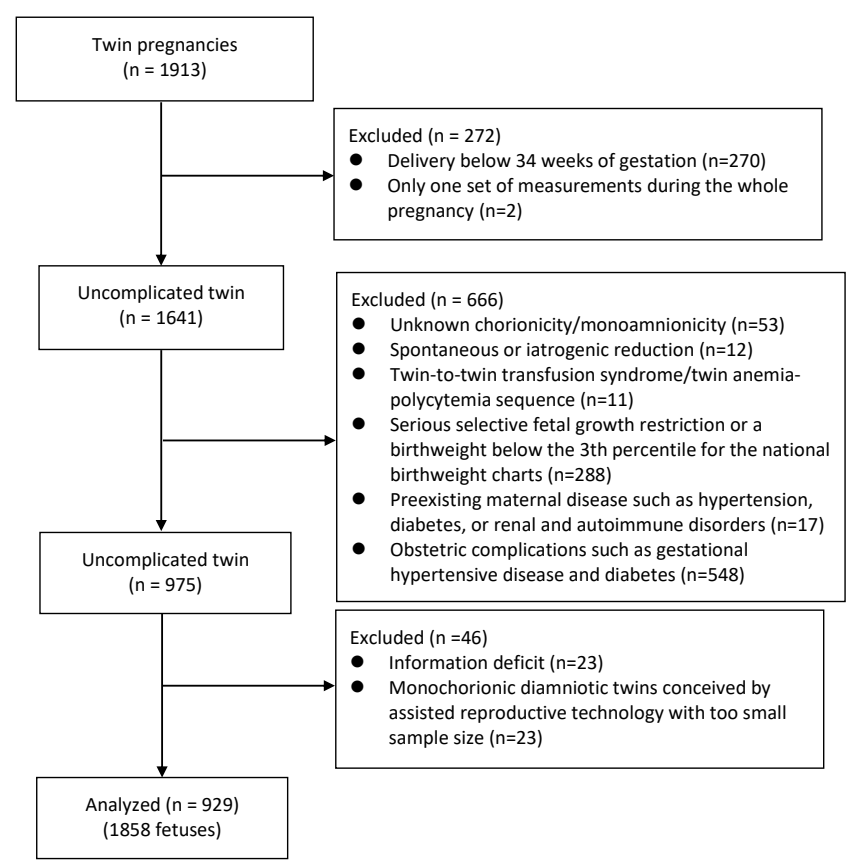



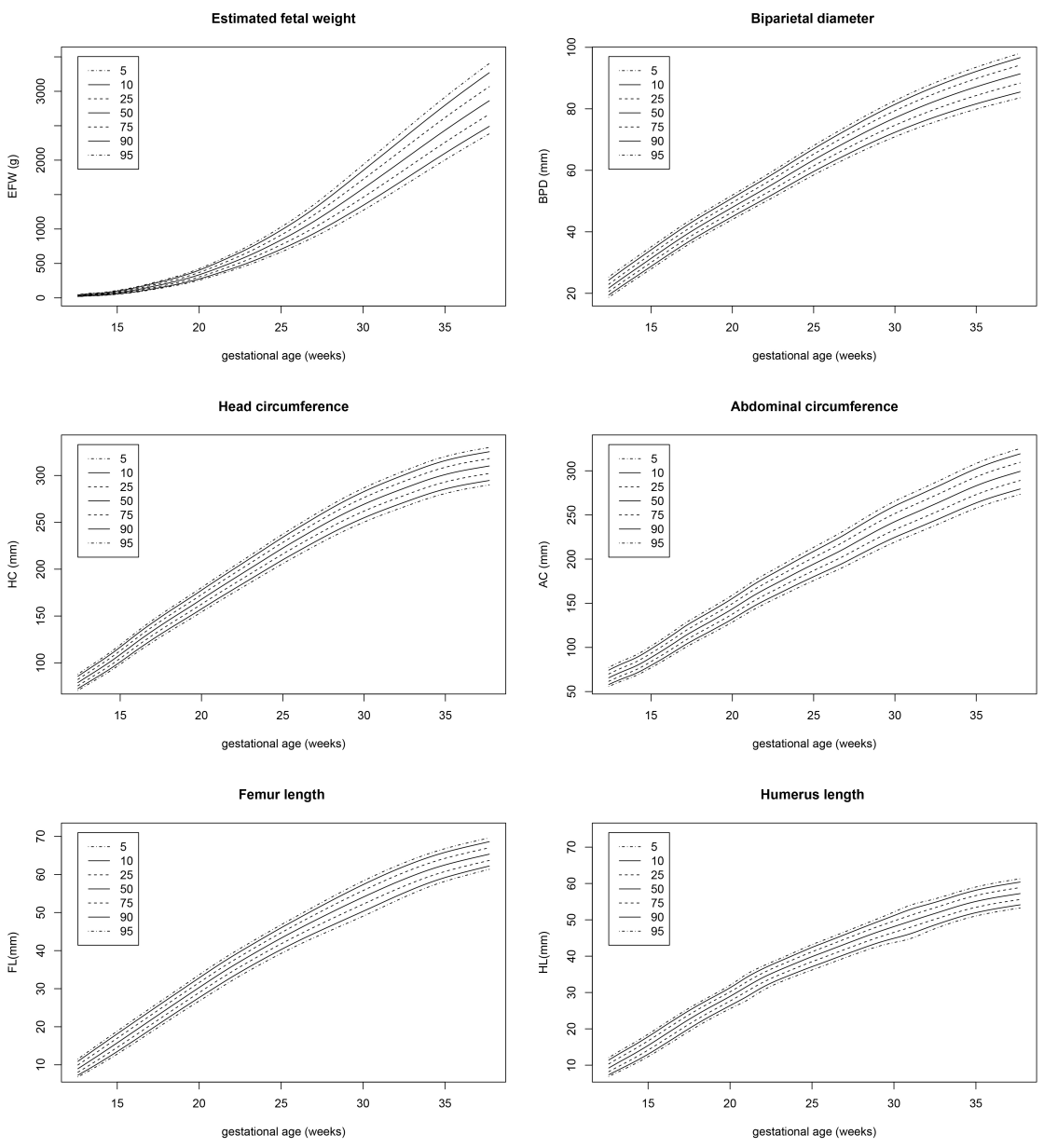

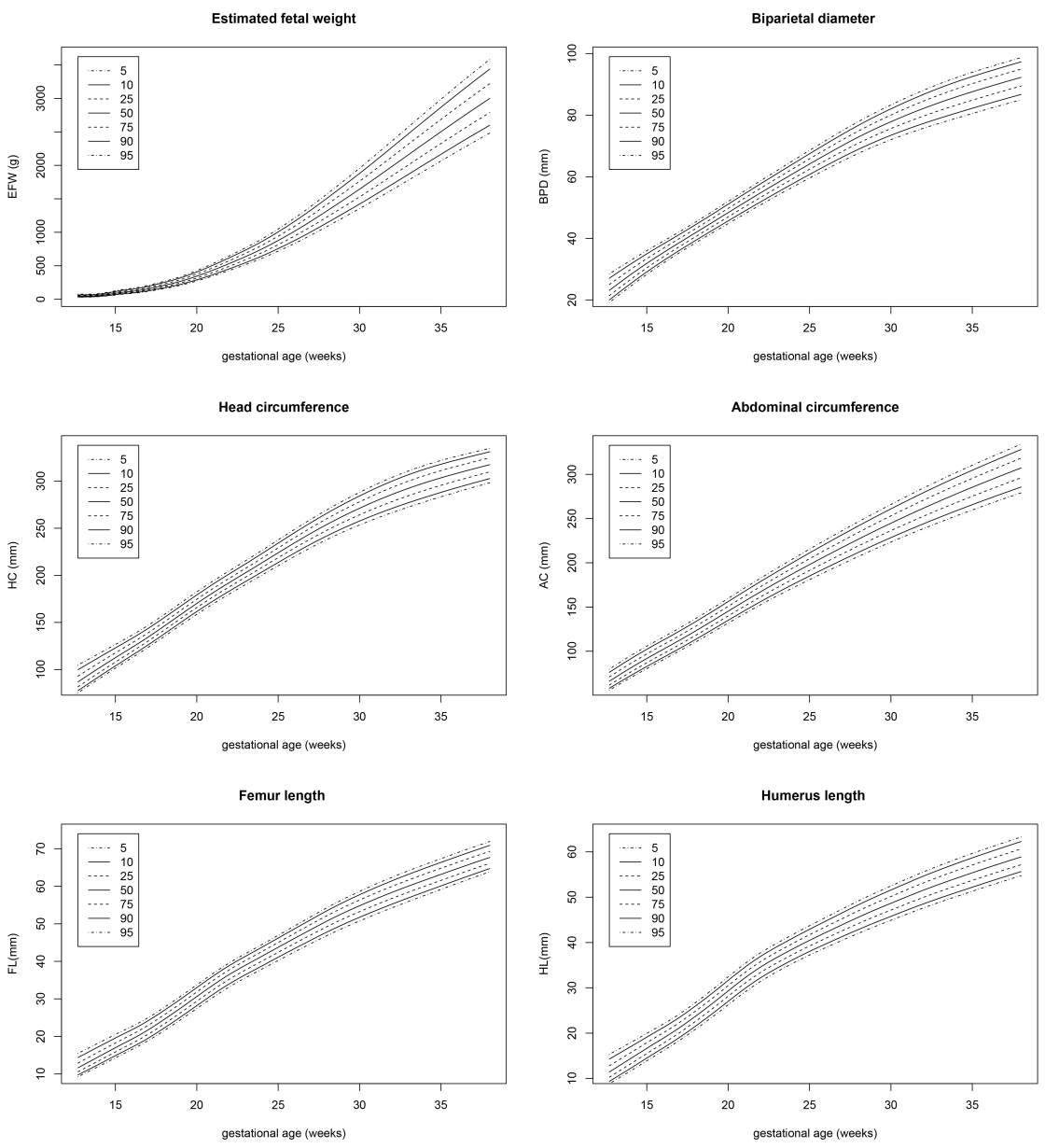

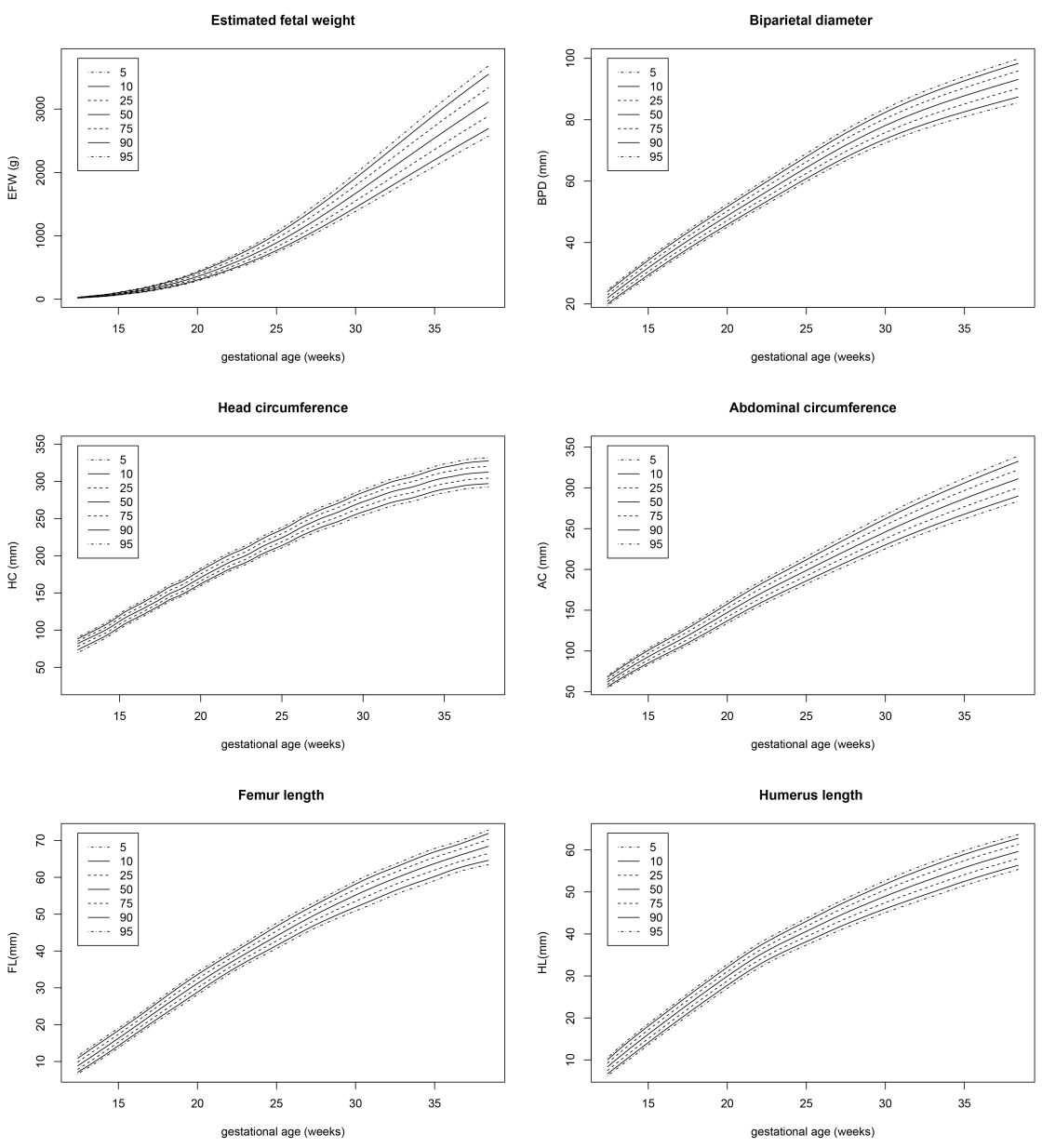